\title{
COUPLED HYDRO-MECHANICAL CRACKING OF CONCRETE USING XFEM IN 3D
}

\author{
Simon-Nicolas Roth*, Pierre Léger ${ }^{\dagger}$ and Azzeddine Soulä̈mani ${ }^{\dagger \dagger}$ \\ *Hydro-Québec \\ 75 boul. René-Lévesque Ouest, Montréal, Québec, Canada, H2Z 1A4 \\ e-mail: roth.simon-nicolas@hydro.qc.ca,www.hydroquebec.com \\ †École Polytechnique de Montréal \\ Department of Civil, Geological and Mining Engineering \\ C.P. 6079, succ. Centre-Ville, Montréal Québec, Canada, H3C 3A7 \\ e-mail: pierre.leger@polymtl.ca,www.polymtl.ca \\ $\dagger^{\dagger}$ École de Technologie Supérieure \\ Department of Mechanical Engineering \\ 1100, rue Notre-Dame Ouest, Montréal, Québec, Canada, H3C 1K3 \\ e-mail: azzeddine.soulaimani@etsmtl.ca,www.etsmtl.ca
}

Key words: Extended Finite Element Method, Continuum Damage Model, Hydrofracturation, Poroelasticity, Drainage

\begin{abstract}
This paper presents a computational method for simulation of 3D hydrofracturation using a segregated and a coupled algorithm hydraulic model. The crack propagation is modeled using a combination of continuous damage and XFEM. The poroelastic problem in the first stage of cracking is modeled using a poro-damage model, where the permeability is linked to the damage coefficient. Once the crack is modeled using XFEM, a hydraulic mesh is automatically generated on the fracture surface with the possibility of including features such as drains. The pressure is computed on the hydraulic mesh taking into account the crack opening computed with the structural model and the different types of flow in a crack: laminar or turbulent, parallel or non-parallel. The pressure computed with the hydraulic mesh is transferred to the structural mesh to recompute the structural response. This procedure, iterated until convergence, can predict hydraulic fracturing taking into account complex flows. A validation example on a wedge-splitting specimen is presented.
\end{abstract}

\section{INTRODUCTION}

The presence of cracks subjected to hydraulic pressures in plain concrete structures is a major concern for their durability, serviceability and stability. To assess the performance of cracked structures several mechanical and hydraulic response parameters have to be computed. This paper presents the development, implementation and application of a new nonlinear strongly coupled finite element hydraulic fracturing model for concrete dams structural stability assessment.

Flow laws in cracks with varying parameters such as aperture, roughness, tortuosity and Reynold's numbers were first established by Louis [10]. Depending on these parameters, the formulation of the hydraulic problem leads to a system of nonlinear equations. The inclusion of drains, with nearby very strong hydraulic gradients, makes it necessary to have 
different (multi-physics) finite element models; one with a hydraulic mesh and one with a mechanical mesh. Therefore, the hydraulic and mechanical subproblems are solved using a partitioned procedure, as they have different resolution requirements and their computational domains have non-matching discrete interfaces. Thus, a key aspect is the transfer of the structural crack apertures to the hydraulic mesh, and the transfer of the hydraulic pressures to the mechanical problem by respecting the applied load equilibrium [6].

In this work, the regularized local anisotropic continuum poroelastic damage method (CPDM) is used to describe the first stage of the fracture process zone (FPZ) formation. When the damage has reached a critical value, a switch to the cohesive XFEM model is achieved by ensuring that the fracture energy that remains to be dissipated by the CPDM model is transferred to the XFEM model [14]. The XFEM formulation makes possible the computation of the crack aperture as well as the application of pressure on the crack surfaces for simulation of hydraulic fracture initiation and propagation. The hydraulic mesh is automatically generated on the crack surface with the possible inclusion of drains. Finally, a crack-tracking technique is used to propagate the crack path along a single row of finite elements.

An application of the proposed hydromechanical constitutive model and numerical solution strategy on a wedge-splitting case illustrates the effectiveness of the model to predict hydrofracturation.

\section{COUPLED HYDRO-MECHANICAL PROBLEM CONSIDERING MATE- RIAL DAMAGE AND DISCONTINU- ITY}

The Biot model of a porous material saturated with a fluid assumes that the solid skeleton is permeated by an interconnected network of pores filled with a moving fluid. The description of stress in the fluid is limited to its hydrostatic component. Therefore, the total stress $\sigma$ in Voigt notation is related to the effective stress $\boldsymbol{\sigma}^{\prime}$ and the liquid pressure $p$ such that:

$$
\boldsymbol{\sigma}=\boldsymbol{\sigma}^{\prime}+\mathbf{m} p
$$

where $\mathbf{m}$ is defined for isotropic behavior as

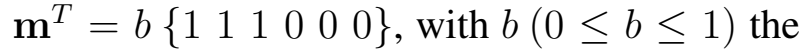
Biot coefficient. Constitutive equations are expressed in terms of balances of momentum and fluid mass for steady state pressure as follows:

$$
\begin{aligned}
& \nabla \cdot \boldsymbol{\sigma}=0 \\
& \nabla \cdot \mathbf{v}=0
\end{aligned}
$$

where $\mathbf{v}$ is the fluid velocity vector. In the presence of a discontinuity, equation (2) is solved using a combined XFEM-damage mechanics approach [14] for which the three dimensional formulation is given in section 3. Equation (3) can be solved either using a segregated or a coupled algorithm. The coupled algorithm is used until transition to XFEM occurs. This transition takes place when the coalescence and ramification of the microscopic cracks form a weak discontinuity. For fluid flow through discontinuities, it is common to make the assumption of a laminar and incompressible flow between two smooth parallel plates. Using a segregated algorithm it is possible to solve the nonlinear flow problem in the discontinuity with less assumptions without affecting the overall solution procedure. The method will be presented in section 4 .

\section{THREE-DIMENSIONAL DISCONTI- NUITY MODEL USING A TRANSI- TION FROM CDM TO XFEM}

A strategy based on a transition from a continuum approach to a kinematic XFEM approach for cohesive crack propagation is used. This process allows the benefits of the continuum damage approach to be combined with the benefits of the cohesive extended finite element method. The regularized local anisotropic continuum damage approach is used to describe the first stage of the FPZ formation. When the damage has reached a critical value, a switch to the cohesive XFEM model is achieved by ensuring 
that the energy that remains to be dissipated by the continuous damage model (CDM) is transferred to the XFEM model. Because anisotropic models are less sensitive to the directional bias of a finite element mesh [9], only the crack tracking algorithm is formulated using a nonlocal theory. The model has the benefit of not adding any additional parameters during the energy transfer between the CDM and the cohesive XFEM models.

\subsection{CONTINUUM DAMAGE MODEL}

The non-linear behavior of concrete under monotonic loading is described using an anisotropic formulation. The behavior of a damaged material results in the constitutive relation of an undamaged material in which the usual stress is replaced by the effective stress by invoking the principle of energy equivalence. The effective stress $\tilde{\boldsymbol{\sigma}}$ is defined as:

$$
\tilde{\boldsymbol{\sigma}}=\mathbf{M}^{-1} \boldsymbol{\sigma}
$$

where $\mathbf{M}$ is the anisotropic damage tensor with the index in each damage direction equal to zero when the material is undamaged, and equal to one when it is completely damaged. With the energy equivalence concept, the local damaged constitutive tensor $\mathbf{C}_{\mathbf{d}}{ }^{(l)}$ is given by:

$$
\mathbf{C}_{\mathbf{d}}{ }^{(l)}=\mathbf{M}^{-1} \mathbf{C}_{\mathbf{0}}\left(\mathbf{M}^{-1}\right)^{T}
$$

The damage tensor is a function of the internal scalars $\kappa_{i}(i \in[1,3])$. This parameter initially equals the damage threshold $r_{0}$ and is the largest recorded value of the principal strains $\varepsilon_{i}$ during the damage process. This evolution is governed by the Kuhn-Tucker condition, given as a loading function $f_{i}$ such that:

$$
f_{i}\left(\varepsilon_{i}, \kappa_{i}\right)=\varepsilon_{i}-\kappa_{i}
$$

Loading is indicated by $f_{i} \geq 0$ and unloading by $f_{i}<0$. The unloading behavior can be simulated using the secant stiffness. Damage is initiated when a tensor norm is greater than the initial threshold $r_{0}$. The tensor norm must take the different behavior of concrete under tension and compression into consideration. One suitable norm that considers the effect of compressive strains $[7,12]$ can be written as follows:

$$
\bar{\varepsilon}=\sqrt{\sum_{i=1}^{3}\left(\left\langle\varepsilon_{i}^{a v}\right\rangle^{2}+m\left\langle-\varepsilon_{i}^{a v}\right\rangle^{2}\right)}
$$

where $\langle\ldots\rangle$ are the Macaulay brackets: $\left\langle\varepsilon_{i}^{a v}\right\rangle=$ $\varepsilon_{i}^{a v}$ if $\varepsilon_{i}^{a v}>0,\left\langle\varepsilon_{i}^{a v}\right\rangle=0$ if $\varepsilon_{i}^{a v}<0$ and $m=\left(\frac{f_{t}}{f_{c}}\right)^{2}$ with $f_{t}$, the tensile strength and $f_{c}$ the compressive strength.

The damage evolution law is the one proposed in [8]:

$$
d=1-\sqrt{\frac{r_{0}}{\bar{\varepsilon}} \exp \left(-R\left(\bar{\varepsilon}-r_{0}\right)\right)}
$$

where $r_{0}=\frac{f_{t}}{E_{0}}$ is the initial threshold defined in terms of the tensile strength and the elastic modulus $E_{0}$. Mesh objectivity requirements are satisfied adequately by introducing a regularization based on the energy equivalence. The stress-strain diagram is adjusted such that the fracture energy is conserved regardless of the size of the element considered. Therefore, the mesh size does not have to respect a maximum size corresponding to the characteristic length of concrete. This is of great importance when analyzing a large structure such as a dam where this dimension is small compared to the size of the dam. Thus, the derivation of $R$ in equation (8) is done in a way that satisfies the mesh objectivity requirements and is defined as:

$$
R=\frac{2 E_{0} f_{t} l_{\text {rve }}}{2 E_{0} G_{F}-f_{t}^{2} l_{\text {rve }}} \geq 0
$$

where $G_{F}$ is the fracture energy and $l_{\text {rve }}$ is the representative volume element characteristic length. To satisfy the requirements that $R \geq$ $0, l_{\text {rve }}$ must be smaller than:

$$
l_{\text {rve }} \leq \frac{2 E_{0} G_{F}}{f_{t}{ }^{2}}
$$

The damage evolution is based on the principal strains exceeding the damage threshold $r_{0}$ :

$$
\text { - if } \kappa_{i}>r_{0} \text { then } d_{i}=d \quad i \in[1,3]
$$


The anisotropic damage tensor can now be defined by:

$$
\mathbf{M}=\left[\begin{array}{cc}
M_{11}^{-1} & 0 \\
0 & M_{22}^{-1}
\end{array}\right]
$$

with

$$
\begin{aligned}
M_{11}^{-1} & =\left[\begin{array}{ccc}
\left(1-d_{1}\right)^{2} & \left(1-d_{1}\right)\left(1-d_{2}\right) & \left(1-d_{1}\right)\left(1-d_{3}\right) \\
\left(1-d_{1}\right)\left(1-d_{2}\right) & \left(1-d_{2}\right)^{2} & \left(1-d_{2}\right)\left(1-d_{3}\right) \\
\left(1-d_{1}\right)\left(1-d_{3}\right) & \left(1-d_{2}\right)\left(1-d_{3}\right) & \left(1-d_{3}\right)^{2}
\end{array}\right] \\
M_{22}^{-1} & =\left[\begin{array}{ccc}
\chi_{12} & 0 & 0 \\
0 & \chi_{23} & 0 \\
0 & 0 & \chi_{13}
\end{array}\right] \\
\chi_{i j} & =\frac{2\left(1-d_{i}\right)^{2}\left(1-d_{j}\right)^{2}}{\left(1-d_{i}\right)^{2}+\left(1-d_{j}\right)^{2}}
\end{aligned}
$$

Damage in either of the principal directions leads to a reduction of the shear resistance by the coefficient $\chi$ similar to that found in smeared crack models. The damage tensor is valid in the local reference frame (aligned with the principal strains directions). Hence, in the global reference frame, this tensor must be rotated by the transformation matrix given by:

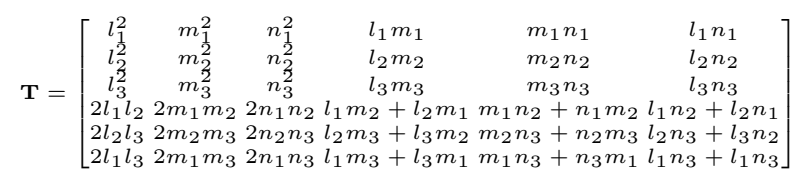

with the direction cosine $\varepsilon_{i}=\left\{l_{i}, m_{i}, n_{i}\right\}$. The transformation from the local to the global reference frame of the damaged constitutive tensor is given by:

$$
\mathbf{C}_{d}^{(g)}=\mathbf{T}^{\mathrm{T}} \mathbf{C}_{d}^{(l)} \mathbf{T}
$$

Finally, the damage is considered to be distributed on a representative volume element $l_{\text {rve }}$. Therefore, this measure is given by the relation:

$$
l_{\mathrm{rve}}=\sqrt[3]{V_{e}}=\sqrt[3]{\sum_{i=1}^{n_{\text {int }}} w_{i} \operatorname{det}_{i}}
$$

with $V_{e}$ the element volume, $n_{\text {int }}$ the number of integration points, $w$ the weight and det the determinant associated with the Gauss point. Similarly, the strains $\varepsilon$ are averaged over the volume and given by:

$$
\varepsilon^{a v}=\frac{\sum_{i=1}^{n_{\text {int }}} \varepsilon_{i} w_{i} \operatorname{det}_{i}}{\sum_{i=1}^{n_{\text {int }}} w_{i} \operatorname{det}_{i}}
$$

These averaged strains are used to evaluate the loading/unloading function (6).

\subsection{COHESIVE XFEM REPRESENTA- TION OF THE DISCONTINUITY}

Within the framework of the extended finite element method, cracks can be modelled by enriching the displacement interpolation of the element crossed by the discontinuity with special purpose functions and by introducing additional unknown $\hat{a}$ to the problem:

$$
u^{h}(x)=\sum_{\forall I} N_{I}(x) \bar{a}_{I}+\sum_{J \in S_{H}} N_{J}(x) \psi(x) \hat{a}_{J}
$$

with $N$ the standard shape functions of the finite element method and $\bar{a}$ the standard degrees of freedom of the problem. The nodal values $\hat{a}$ are the additional degrees of freedom that adjust the enrichment so that they approximate the function $\psi(x) . S_{H}$ represents the set of nodes that are enriched because the discontinuity passes through the element to which they belong. To represent a strong discontinuity such as a crack, the Heaviside function $(\mathscr{H})$ can be used for $\psi(x)$ :

$$
\psi(x)=\left[\mathscr{H}(f(x))-\mathscr{H}\left(f\left(x_{J}\right)\right)\right]
$$

with $x_{J}$ the position of node $J$. The enrichment function is shifted so that the product of the shape function $N_{J}$ and the enrichment function cancels out at each node. Therefore, only those elements that are crossed by the discontinuity should be treated differently. To account for the additional cohesive forces transferred through the crack, the weak form of equation (2), taking into account the concept of effective stress given in equation (1) and using test functions $(\delta \bar{u}, \delta \hat{u})$, is defined as:

$$
\begin{array}{r}
\int_{\Omega} \nabla \delta \bar{u}: \boldsymbol{\sigma} \mathrm{d} \Omega+\int_{\Omega^{\star}} \nabla \delta \hat{u}: \boldsymbol{\sigma} \mathrm{d} \Omega^{\star} \\
+\int_{\Gamma_{d}} \delta \hat{u} t(\llbracket \mathbf{u} \rrbracket) \mathrm{d} \Gamma_{d}=\int_{\Gamma} \delta \bar{u} \mathbf{t}_{p} \mathrm{~d} \Gamma \\
+\int_{\Gamma_{d}} \delta \hat{u} \mathbf{t}_{d} \mathrm{~d} \Gamma_{d}
\end{array}
$$


where $t_{p}$ is the prescribed traction on the external boundary, $\mathbf{t}_{d}$ the prescribed traction in the discontinuity (pressure computed in section 4.2), $t(\llbracket \mathbf{u} \rrbracket)=f_{t} e^{-\frac{f_{t} \llbracket \mathbf{u} \rrbracket}{G_{F}}}$ the cohesive force, $\Omega^{\star}$ has been introduced, so that the integration is performed by parts due to the Heaviside function. With the boundary conditions:

$$
\boldsymbol{\sigma} \mathbf{n}_{\Gamma}=\mathbf{t}_{p}, \quad \mathbf{u}=\mathbf{u}_{p}
$$

$\mathbf{n}_{\Gamma}$ being the outward unit normal on the external boundary and $\mathbf{t}_{p}, \mathbf{u}_{p}$ respectively the prescribed external traction and displacements yields equation to be solved for the mechanical problem with the cohesive XFEM representation of the discontinuity. Note that when the element is not enriched and the continuous damage model is used, the terms on $\delta \hat{u}$ vanish.

\subsection{TRANSITION FROM CONTINUOUS DAMAGE MECHANICS TO COHE- SIVE XFEM}

Following the approach proposed in [14], conservation will be enforced using mode I energy dissipation. The three conditions required for the conservation of energy during transition are:

- The energy dissipation of the continuous damage mechanics approach from damage initiation until the transition strain occurs must be equivalent to the energy dissipated by the cohesive model from damage initiation until the transition opening is reached;

- The energy remaining to be dissipated from the transition strain in the CDM model to complete material separation must be equal to that of the cohesive model;

- The initial traction stress of the cohesive surface must be equal to the CDM model stress at the transition strain.

This energy conservation is applied using the cohesive force. The transition from CDM to XFEM is achieved when $d>d_{\text {crit }}$. According to [14], $d_{\text {crit }}$ is chosen such that $0.5 \leq d_{\text {crit }} \leq$ 0.7 .

\section{COUPLED HYDRO-MECHANICAL PROBLEM}

The transport process with regard to the averaged motion of the fluid involves molecular diffusion. These molecular diffusion and viscous flow are both dissipative. Positivity of the dissipation associated with the viscous flow of the fluid through the porous solid can be written in the form $(-\nabla p \cdot v \geq 0)$ [4]. The fluid movement is governed by the law relating the velocity vector $\mathbf{v}$ to the driving force $-\nabla p$ producing the flow. Linearly relating $\mathbf{v}$ to $-\nabla p$ and neglecting body loads is the simplest form that this law can take and leads to the expression of Darcy's law:

$$
\mathbf{v}=-\mathbf{K} \nabla p,
$$

with $\mathbf{K}=\delta_{i j} k / \mu$ the permeability coefficient matrix, $k$ is the intrinsic permeability and $\mu$ the fluid viscosity. Hence, the fluid transport in the interstitial space is described by replacing the velocity in the continuity equation (3) by Darcy's law leading to:

$$
\nabla \cdot(-\mathbf{K} \nabla p)=0
$$

Using $\delta p$ as the test function, the weak form of this equation:

$$
-\int_{\Omega}(\nabla \delta p \mathbf{K} \nabla p) \mathrm{d} \Omega=\int_{\Gamma} F \delta p \mathrm{~d} \Gamma
$$

is added to equation (20) for complete mathematical description of the poroelastic model with the prescribed pressure $p_{p}$ boundary condition:

$$
p=p_{p} \text { on } \Gamma_{p}
$$

It is assumed that there is no membrane effect within the crack, therefore the pressure term is not enriched and the pressure field is continuous across the discontinuity surface.

In addition to the interstitial space, this form of the continuity equation will be expanded for two other types of flow that occurs: flow in the damaged material with the presence of microcracks and flow in macrocrack (discontinuity). 


\subsection{PORO-DAMAGE MODEL}

A parallel between material damage and increasing permeability in the porous flow model can be achieved. Indeed, damaged concrete has a large number of pores that can be filled with the liquid phase. This observation may be represented mathematically by modifying the permeability and/or the Biot coefficient as a function of the damage level.

According to [13] changes in concrete permeability can be split in two parts. In the first part, when microcracking linked to low-level damage (0 to $15 \%$ ) take place, and secondly, when macro-crack occurs beyond the peak load. Based on experimental results, this reference gives a damaged-permeability relationship in the prepeak phase of concrete (valid for damage values between 0 and 0.18 ) as an exponential function:

$$
k_{D}=k_{0} \exp \left(\left(\alpha d_{1}\right)^{\beta}\right)
$$

with $\alpha=11.3, \beta=1.64$ and $d_{1}$ the first principal tensile damage scalar. For strong damage, the permeability of a crack is given by HeleShaw flow (equation (31)) (also named planePoiseuille flow). A single mathematical law, based on the law of mixtures, was proposed in [5] which allows to describe the evolution of permeability from the initiation of microcracks until the opening of the macrocrack.

$$
\log \left(k_{m}\right)=\left(1-d_{1}\right) \log \left(k_{D}^{F}\right)+d_{1} \log \left(k_{c r}\right)
$$

with $k_{D}^{F}$ a limited Taylor expansion of the exponential relation. Assuming that the permeability change in the damaged material is isotropic, the permeability matrix from equation (24) is given by $\mathbf{K}=\delta_{i j} k_{m} / \mu$. Similarly, the Biot coefficient increases such that:

$$
b=b_{0}+\left(1-b_{0}\right) d_{1}^{2}
$$

with $b_{0}$ the initial Biot coefficient.

\subsection{FLOW IN DISCONTINUITIES}

Starting with the Navier-Stokes equations and using the assumptions that the flow through the crack wall is zero, that the inertial forces are negligible compare to the viscous forces and that the flow is steady state one can derive the general equation for flow in the crack as:

$$
\nabla \cdot\left(-w_{c r} k_{c r} \nabla H\right)=0
$$

$w_{c r}$ is the crack opening and the total water level is given by:

$$
H=z+\frac{p}{\rho g}+\frac{\mathbf{v}_{c r}^{2}}{2 g}
$$

with $\mathbf{v}_{c r}$ the velocity of the fluid in the crack, $g$ the gravity, $\rho$ the fluid density. The hydraulic conductivity coefficient $k_{c r}$ is related to the flow profile inside the crack. For example, for a parallel laminar flow where the velocity profile is parabolic, the hydraulic conductivity coefficient is given as:

$$
k_{c r}=\frac{g w_{c r}^{2}}{12 \nu}
$$

corresponding to the Hele-Shaw flow, with $\nu$ the fluid kinematic viscosity. For other types of flow profiles, [10] has recorded the results of several authors and created a series of five flow laws with their associated ranges of validity. A parameter $h_{a} / D_{h}$ refered to as the "relative roughness" is used to define the crack roughness, where $h_{a}$ is the average asperity height in the crack and $D_{h}$ is the hydraulic diameter (equals two time the crack opening). By definition, the relative roughness of a crack is zero for a smooth crack and 0.5 for a rough crack with asperities the size of the crack opening. Flow in the crack is considered parallel for low values of relative roughness $\left(h_{a} / D_{h}<0.033\right)$, whereas for $h_{a} / D_{h} \geq 0.033$, the flow will be nonparallel. The flow velocity distribution made by Louis, taking into account the roughness of the crack is given by:

$$
\mathbf{v}_{c r}=k_{c r}|\nabla H|^{\alpha-1} \nabla H
$$

The values of hydraulic conductivity $k_{c r}$ and the exponent $\alpha$ depend on the type of flow as described in Figure 1. For hydraulically smooth parallel flow the transition from laminar to turbulent flow occurs at approximately $R_{e}=2300$ 


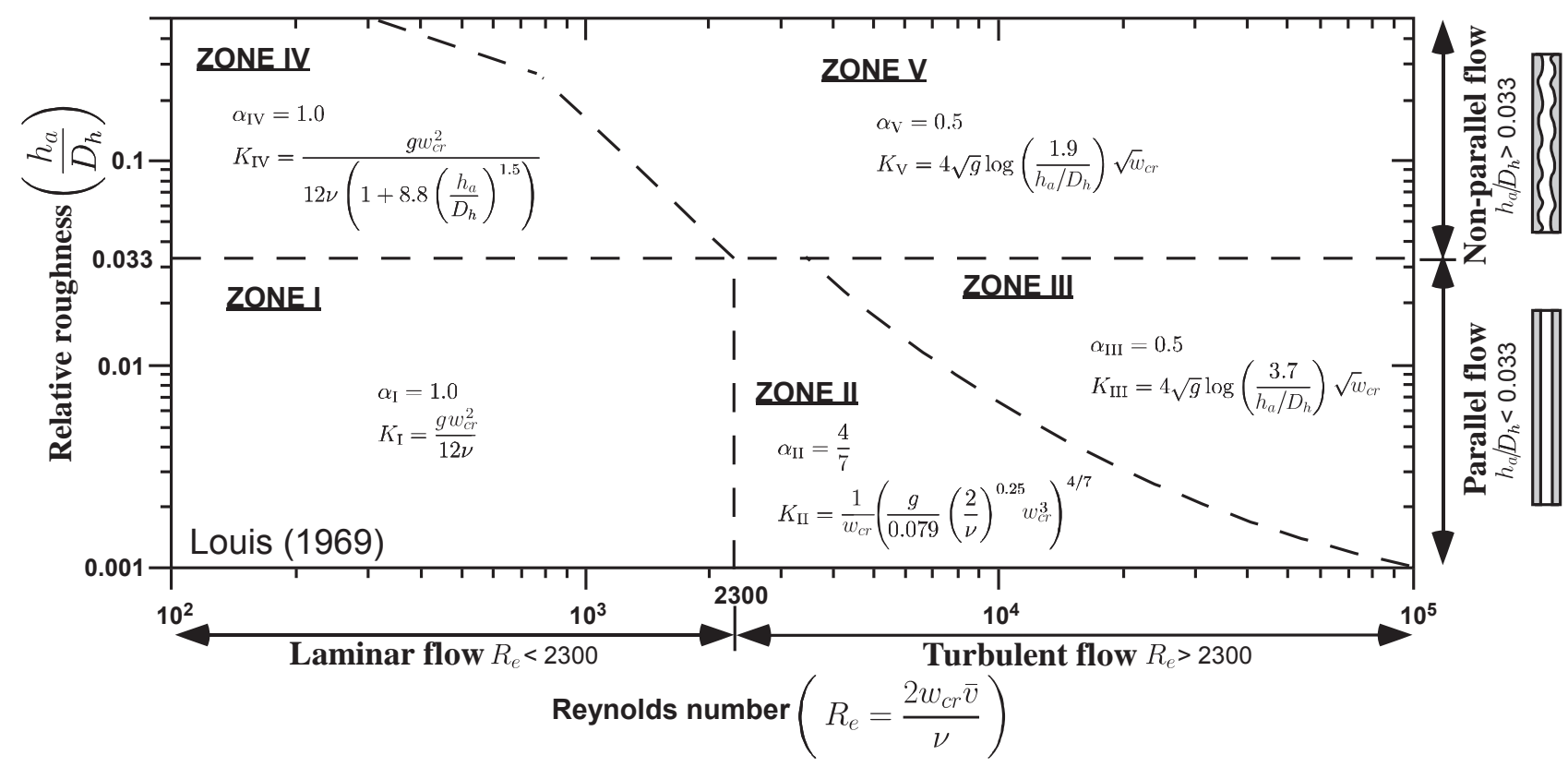

Figure 1: Compilation of the different flow laws and their range of validity [after [10]]

with the Reynolds number defined by:

$$
R_{e}=\frac{2 w_{c r}\left\|\mathbf{v}_{c r}\right\|}{\nu}
$$

Adding boundary conditions (water head $h$, at the boundaries including the drain, $H_{d}$, or impervious condition), equation (29) enables to solve the flow problem in the discontinuity. If turbulent flow conditions are encountered (zones II, III, and V), the velocity is not proportional to the hydraulic gradient and the formulation of the problem leads to a system of non-linear equations.

Defining an open set $\Omega$ in the space $\mathbb{R}^{2}$ where one seeks to solve equation (29) discretized into a collection of linear T3 triangular elements. The mesh is in the $\mathbb{R}^{2}$ space only locally, namely, it is on a surface in space $\mathbb{R}^{3}$, but when solving the problem, the element is transferred to a local coordinate system in the $\mathbb{R}^{2}$ space. The use of linear T3 triangle simplifies the evaluation of the conductivity matrix in the local coordinate system as it ensures that the element is planar. Because the total hydraulic load is independent from the coordinate system used (similar to a potential function), it is not necessary to transpose the conductivity matrix in the global coordinate system. Using this type of formulation, care must be taken to add the effect of total water head as a function of discontinuity surface elevation variation.

\subsection{COUPLING OF FLUID-STRUCTURE INTERACTION WITH NON- MATCHING INTERFACE}

When the interface mesh between the structural and hydraulic subproblems is identical (each nodes on the hydraulic mesh has an equivalent node located at the same position on the surface of the structure), the transfer of pressures and crack aperture is a trivial operation. However, these subproblems have different resolution requirements and their computational domains have non-matching discrete interfaces. Therefore, the hydraulic-structure interface must be coupled. A suitable method is to make the variable transfer taking into account the interpolation functions used by the finite elements as proposed in [6]. The first step is to find for each hydraulic node in which structural element the hydraulic node (or its projection on the structural element) is located. Once the element is found, the second step is to define the interpolating function. Note if the discontinuity intersection with the structural hexahedral element yields a quadrangular Q4 element, the el- 
ement is divided in two T3 triangular elements. Using barycentric coordinates computation, one can perform these two steps at once. Hence, the computation of the barycentric coordinates for the projection $P^{\prime}$ of a point $P$ into the plane of a triangle defined by segments $\vec{u}, \vec{v}$ and passing by point $Q$ can be computed as:

1. Compute the triangle normal: $\vec{n}=\vec{u} \otimes \vec{v}$

2. Compute vector between point $P$ and $Q$ : $\vec{w}=P-Q$

3. Compute the barycentric coordinates:

$$
\begin{aligned}
& N_{1}=(\vec{u} \otimes \vec{w}) \odot \vec{n} /(\vec{n} \odot \vec{n}) \\
& N_{2}=(\vec{w} \otimes \vec{v}) \odot \vec{n} /(\vec{n} \odot \vec{n}) \\
& N_{3}=1-N_{2}-N_{1}
\end{aligned}
$$

4. Point $P$ is inside the triangle if: $0 \leq$ $N_{1} \leq 1$ and $0 \leq N_{2} \leq 1$ and $0 \leq N_{3} \leq 1$

The hydraulic node crack aperture $w_{c r}^{h}$ can be interpolated from the associated structural triangular element crack aperture $w_{c r}^{i=1 . .3}$ with the interpolation function computed in step 3:

$$
w_{c r}^{h}=\sum_{i=1}^{3} N_{i} w_{c r}^{i}
$$

The inverse procedure is applied for interpolating the hydraulic pressure computed in section 4.2 to the structural mesh.

\section{SOLUTION ALGORITHM FOR DIS- CONTINUITY PATHS COMPUTA- TION}

Following the global method proposed in [11], we are looking for isosurfaces of a scalar field $\theta$ tangent to a direction field such that it satisfies the conditions $\mathbf{T} \nabla \theta=0$ and $\mathbf{S} \nabla \theta=0$. Therefore it is necessary to solve the heat transfer equation with adiabatic boundary conditions and no internal heat source:

$$
\begin{gathered}
\nabla \cdot \mathbf{q}=0 \text { in } \Omega \\
\mathbf{q}=-\mathbf{K}_{p} \nabla \theta \text { in } \Omega \\
\mathbf{q} \cdot \mathbf{n}=0 \text { on } \Gamma_{q}
\end{gathered}
$$

$$
\theta=\theta_{p} \text { on } \Gamma_{\theta}
$$

The anisotropic conductivity matrix is given by:

$$
\mathbf{K}_{p}=\mathbf{T} \otimes \mathbf{T}+\mathbf{S} \otimes \mathbf{S}+\zeta \mathbf{I}
$$

with $\zeta$ a small perturbation term $\left(10^{-6}\right)$ to avoid matrix singularity and $\mathbf{S}, \mathbf{T}$ are the eigenvectors perpendicular to the eigenvector of the largest stress eigenvalue. These eigenvalues/eigenvectors are computed using the nonlocal stress tensor. The isosurfaces of the scalar field computed are "potential" discontinuity surfaces. Once the discontinuity evolves, boundary conditions on the nodes attached to the elements crossed by the discontinuity are imposed such that the scalar field is fixed around the discontinuity so that it cannot change position and direction.

The isosurface origin is assumed to be located at the center of an element face located on a boundary. If more than one face is in contact with the boundaries, the centroid of the element is selected as the origin. Using the finite element shape functions, the scalar $\theta_{\text {iso }}$ must be evaluated at this origin. Finally, the signed distances (level-sets) between the discontinuity surface and the nodes are computed with $\theta_{i}-\theta_{i s o}, \theta_{i}$ being the nodal scalar. The crack surface within hexahedral elements are not necessarily planar, hence proper integration method must be used to integrate $\Omega^{\star}$ and $\Gamma_{d}$ in equation (20).

\section{GLOBAL ALGORITHM DETAILS}

Figure 2 gives with the UML activity diagram the global solution algorithm. Step 1 is the resolution of the system of equations (20) and (24). Next, for every elements, the cohesive force is updated if the element is enriched. If the element is not enriched, the constitutive matrix is updated (section 3.1) and the permeability matrix is modified (section 4.1) if the principal strains exceeds the damage threshold $r_{0}$. In step 6, the discontinuity paths are computed (section 5). If the solution has converged, for each element, a check is made to see whether the damage threshold $d_{c r i t}$ is exceeded. If the 


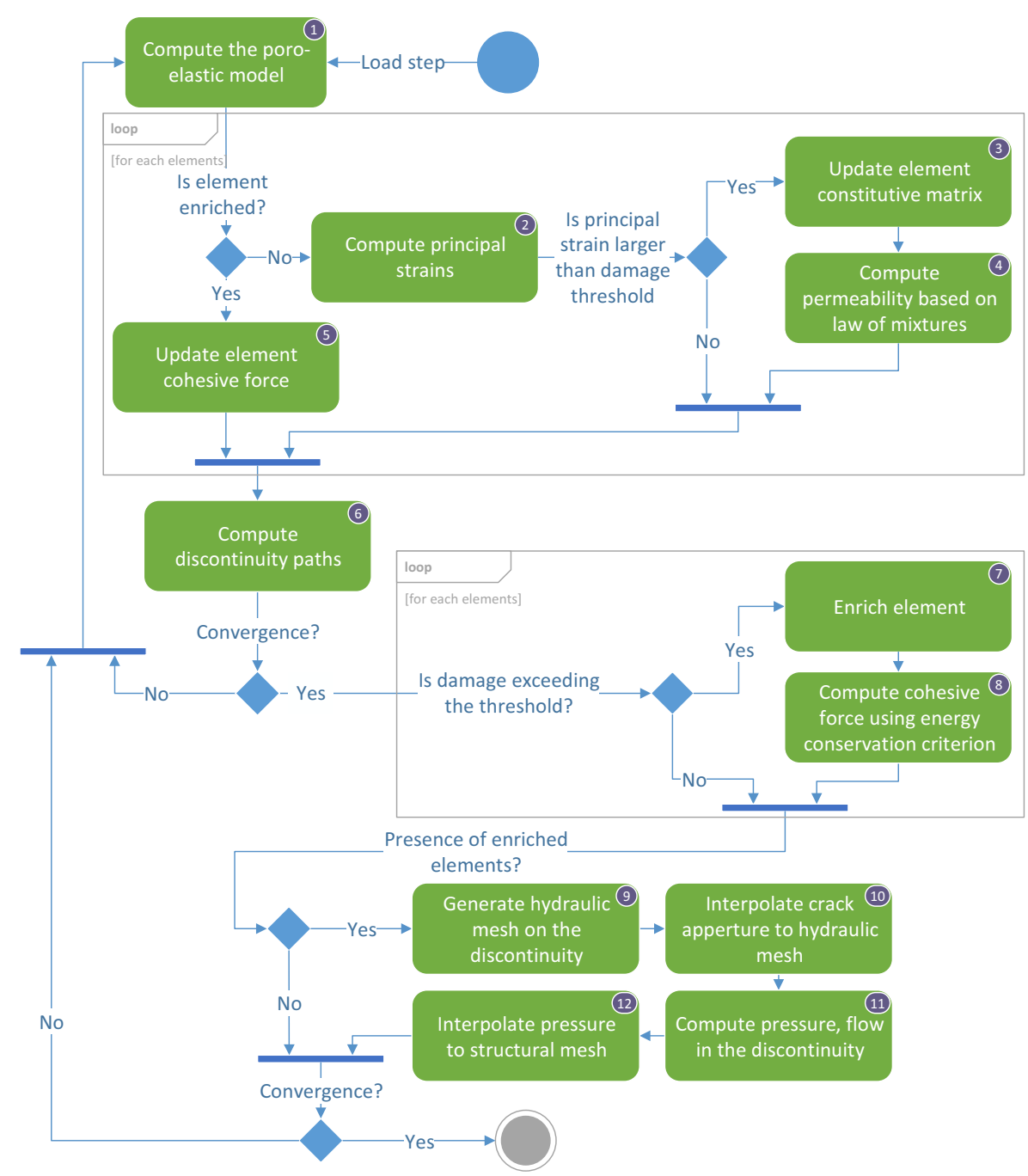

Figure 2: UML activity diagram of the algorithm for one load step

element is not enriched and the threshold is reached, enrich the element. Using the criteria given in section 3.3 the cohesive law for mode I energy conservation is computed during transition from CDM to XFEM. Step 9 to 12 are performed only if the model has enriched elements. In step 9, using a surface reconstruction method [1] on the points located at the intersection of the discontinuities and the mesh, the hydraulic mesh is generated. At this time, any features such as drains must be inserted in the hydraulic mesh. Step 10 involves the coupling of the structural mesh with the hydraulic mesh (section 4.3). Once the apertures are transferred to the hydraulic mesh, solution of the flow in the discontinuity is computed (section 4.2). In step 12 , the pressure computed are transferred to the structural mesh. Finally, if the convergence criterion is verified, compute next load step, else repeat the procedure starting at step 1 .

\section{VALIDATION EXAMPLE}

The wedge-splitting device tested in [3] and simulated numerically in [2] is used as a validation example of the algorithm. The geometry and material properties are given in Figure 3 and Table 1. Five cases will be modeled to show the benefits of the proposed method. The first case (case 1) considers only the continuous damage model without using the XFEM. Hence, only porodamage is used to simulate the hydrofracturation. In the four other cases, the discontinuity is imposed using level-sets and cohesive XFEM is used. Case $2 \mathrm{a}$ has no 
drainage. Case $2 \mathrm{~b}$ has one $20 \mathrm{~mm}$ diameter drain located at the center of the wedge thickness, $30 \mathrm{~mm}$ away from the crack entrance (Figure 4a). Case $2 \mathrm{c}$ has two $20 \mathrm{~mm}$ diameter drains located $20 \mathrm{~mm}$ away from the crack entrance (Figure 4b). Finally, case 3 considers only the pressure applied in the red zone (Figure 3 ) of the wedge without considering poro-elastic effects. To simulate hydrofracturation, the external pressure load applied in the red zone and the pressure boundary condition applied on the nodes in contact with the red zone are increased as a function of the crack mouth opening displacement (CMOD).

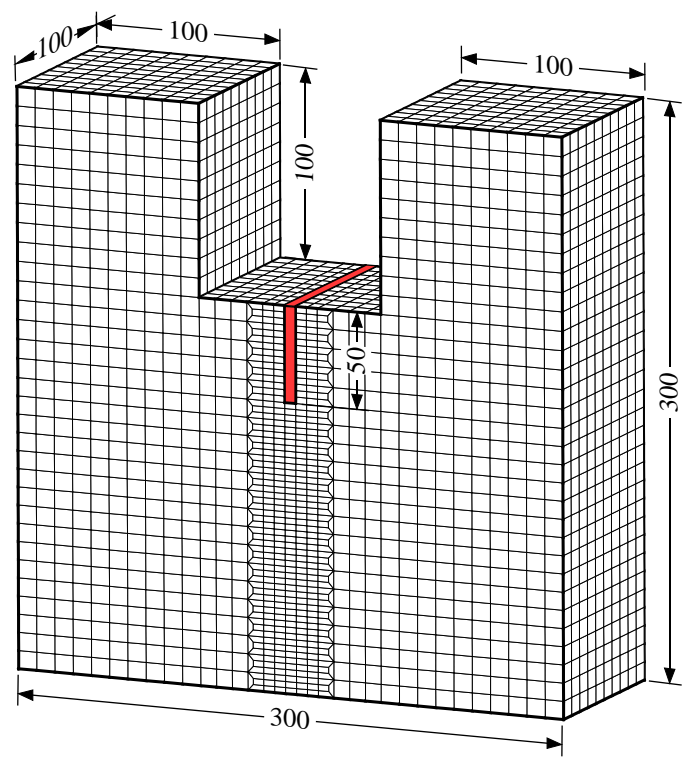

Figure 3: Wedge-splitting finite element model (dimensions in $\mathrm{mm}$ )

Table 1: Material properties for the wedgesplitting specimen

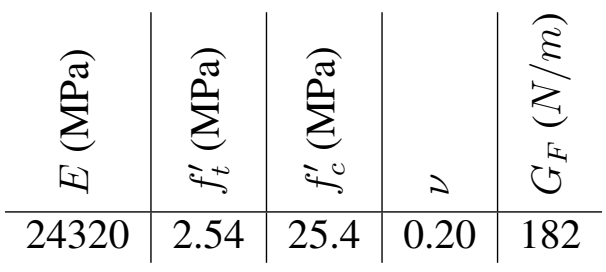

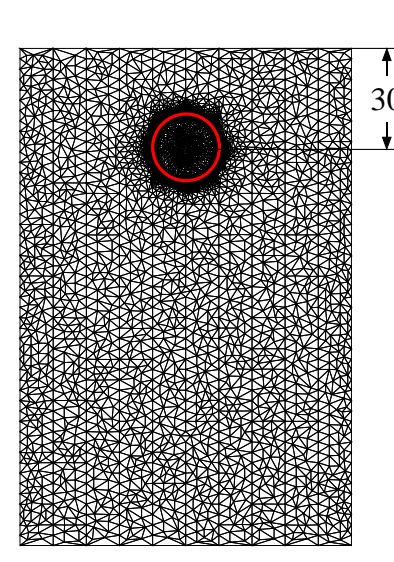

(a)

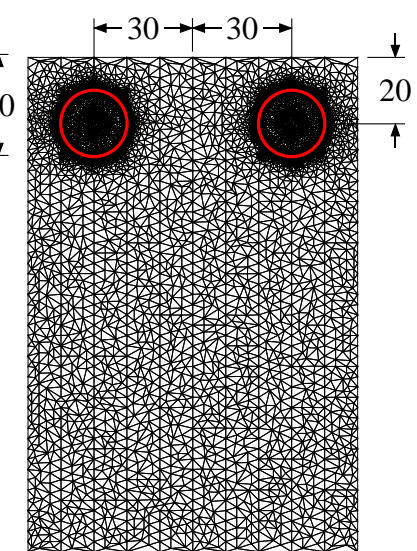

(b)
Figure 4: (a) Drain location for case 2b, (b) Drains locations for case $2 \mathrm{c}$

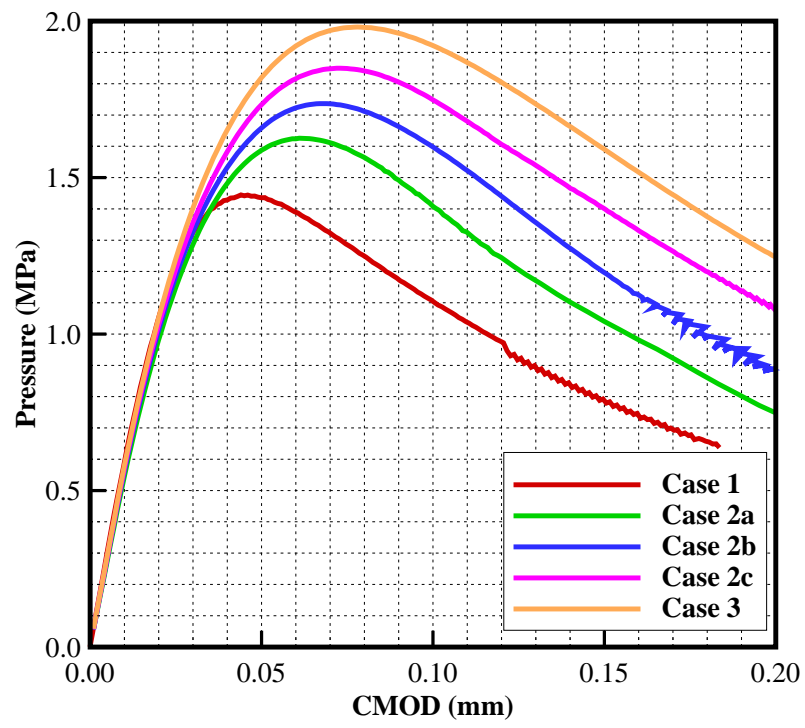

Figure 5: Maximum pressure load as a function of CMOD

Figure 5 gives the pressure load as a function of the CMOD. With a maximum pressure of $1.45 \mathrm{MPa}$, case 1 gives similar results to [2] where the maximum pressure found is 1.30 $\mathrm{MPa}$. It is expected that case $2 \mathrm{a}$ gives results in the same range. However the pressure computed is larger to that of case $2 \mathrm{a}$ by a margin of $0.15 \mathrm{MPa}$. The cause if this margin must be further investigated. The effectiveness of the drainage is proven when comparing this result with those of case $2 b$ and $2 c$. Using two drains give results that are close to case 3 where the 
pressure inside the crack is not considered when crack propagation occurs. Instability in the solution procedure occurs for case 1 and case $2 b$ with CMOD values beyond $0.12 \mathrm{~mm}$. Figure 6 , compares the pressure distribution of cases $2 b$ and $2 \mathrm{c}$ in the cracking surface. When using two drains, the pressure almost drops to zero beyond the drains. This explains the larger pressure response found for this case.

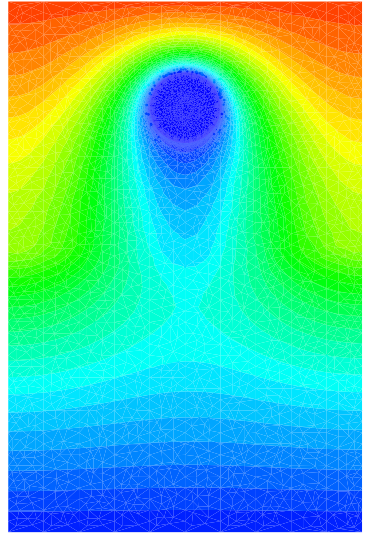

(a)

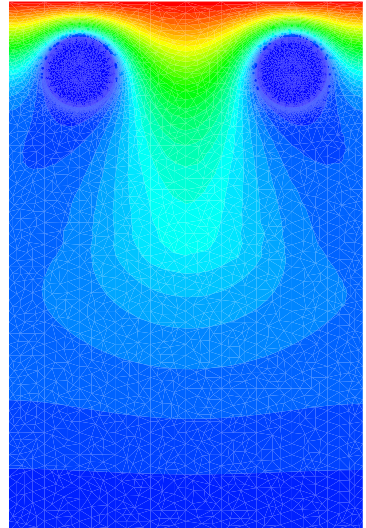

(b)

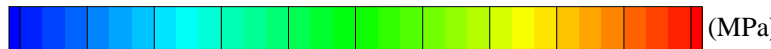

$\begin{array}{lllllllllll}0.00 & 0.18 & 0.36 & 0.54 & 0.72 & 0.90 & 1.08 & 1.26 & 1.44 & 1.62 & 1.80\end{array}$

Figure 6: Pressure distribution on the hydraulic mesh for (a) case $2 b$, (b) case $2 c$

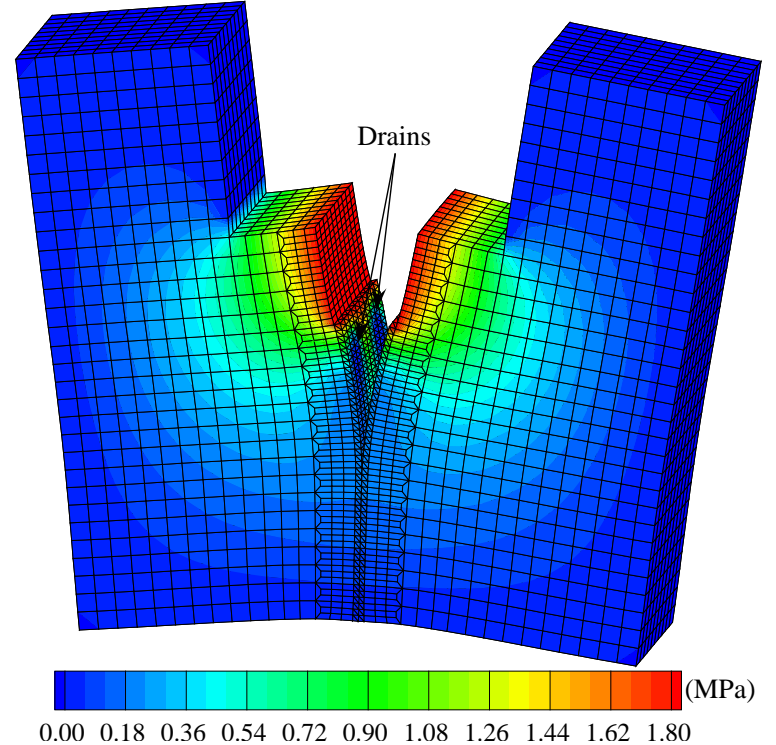

Figure 7: Pressure distribution at maximum pressure load for case 2c
Figure 7 gives the pressure distribution on the structural mesh of the wedge specimen for case 2c. The interpolated pressure boundary condition obtained from the hydraulic mesh are clearly visible. The arrows in the figure are added to emphasize the location of the drains.

\section{CONCLUSION}

The proposed model has shown its ability to model hydrofracturation taking into account complex flows and drainage. This model can be used to assess the performance of cracked hydraulic structures. The inclusion of drainage in complex dam safety assessment can help reduce or even avoid rehabilitation work and thereby lower their costs. The future steps is to validate the complete combined CDM-XFEM algorithm on a $3 \mathrm{D}$ non-planar case.

\section{REFERENCES}

[1] CGal, Computational Geometry Algorithms Library, . http://www.cgal.org.

[2] S. Bhattacharjee, F. Ghrib, R. Tinawi, and $\mathrm{P}$ Léger. Poro-fracture analysis of concrete using a damage mechanics model. pages 1057-1066. 2nd International Conference on Fracture Mechanics of Concrete and Concrete Structures, 1995.

[3] E. Bruhwiler and V. E. Saouma. Water Fracture Interaction in Concrete-Part 1: Fracture Properties. Materials Journal, 92(3):296-303, 1995.

[4] O. Coussy. Mechanics and Physics of Porous Solids. John Wiley \& Sons, Ltd, 2010.

[5] F. Dufour. Contributions à la modélisation numérique de la fissuration des structures en béton avec prise en compte du fluage et estimation de la perméabilité. Technical report, 2007.

[6] C. Farhat, M. Lesoinne, and P. Le Tallec. Load and motion transfer algorithms for fluid/structure interaction problems with 
non-matching discrete interfaces: Momentum and energy conservation, optimal discretization and application to aeroelasticity. Computer Methods in Applied Mechanics and Engineering, 157(1-2):95114, apr 1998.

[7] F. Ghrib and R. Tinawi. Nonlinear Behavior of Concrete Dams Using Damage Mechanics. Journal of Engineering Mechanics, J. Eng. Mech., 121, 1995.

[8] R. M. Gunn. Non-linear analysis of arch dams including an anisotropic damage mechanics based constitutive model for concrete. $\mathrm{PhD}$ thesis, University of Brighton, U.K., 1998.

[9] M. Jirásek and T. Zimmermann. Analysis of Rotating Crack Model. Journal of Engineering Mechanics, J. Eng. Mech., 124(8):842-851, August 1998.

[10] C. Louis. A Study of Groundwater Flow in Jointed Rock and Its Influence on the Stability of Rock Masses. Imperial College Rock Mech. Res. Rep 10, Imp. Coll., London, September 1969.
[11] J. Oliver, A. E. Huespe, E. Samaniego, and E. W. V. Chaves. On strategies for tracking strong discontinuities in computational failure mechanics. Proc.World Congress on Computational Mechanics, Vienna University of Technology, 2002. WCCM V, In H.A. Mang, F.G. Rammerstorfer, and J. Eberhardsteiner.

[12] M.H.J.W. Paas, P.J.G. Schreurs, and W.A.M. Brekelmans. A continuum approach to brittle and fatigue damage: Theory and numerical procedures. International Journal of Solids and Structures, 30(4):579-599, 1993.

[13] V. Picandet, A. Khelidj, and G. Bastian. Effect of axial compressive damage on gas permeability of ordinary and highperformance concrete. Cement and Concrete Research, 31(11):1525-1532, 2001.

[14] S-N. Roth, P. Léger, and A. Soulaïmani. A combined XFEM-damage mechanics approach for concrete crack propagation. Computer Methods in Applied Mechanics and Engineering, 283:923-955, 2015. 